\title{
Neurological Complications of Open Heart Surgery
}

Soon after the development of cardiopulmonary bypass procedures for open heart surgery, reports began to appear indicating that neurological complications occurred frequently [1]. Despite refinements in the apparatus used for cardiopulmonary bypass and improvements in the intraoperative techniques since that time, neurological disorders continue to be reported. The types of abnormalities described include stroke, seizures, hypoxic encephalopathy, delirium, specific disorders of higher cerebral function, and visual field defects [1-4]. Many studies also mention focal motor and sensory disorders, peripheral neuropathy, and brachial plexus injury [4]. Other symptoms considered to be psychiatric in nature include insomnia, anorexia, depression, hostility, disorientation, hallucinations, and delusions [2].

The reported incidence of the neurological complications of open heart operations varies widely depending upon the type of study, ranging from 7 to $61 \%$ for transient and from 1.6 to $23 \%$ for permanent complications [2]. As might be expected, the incidence is much higher in prospective $(28.7 \%$ incidence in a literature review of 804 patients) than in retrospective (11.9\% incidence in a literature review of $1,787 \mathrm{pa}$ tients) studies [2]. Even among prospective studies, however, the incidence varies substantially. In one study of 204 patients, for example, the incidence of neurological complications was $16.2 \%$ for transient and $6.4 \%$ for persisting abnormalities [2]. In another study of 312 patients, however, transient complications occurred in 61\% [4]. Despite the high incidence of transient complications in this latter study, the number of patients with persisting neurological impairments was small [4]. Neurological complications resulted in death in 1 patient $(0.3 \%)$, and severe disability occurred in $4(1.3 \%)$. Forty-eight patients were mildly disabled during the immediate postoperative interval, and the remaining 138 with neurological abnormalities had no serious functional disability [4].

Retrospective evaluations give considerably lower rates of postoperative neurological complications than careful prospective assessments. In a recent retrospective study, for example, a review of 1,669 patients who underwent coronary artery bypass graft surgery showed 75 cerebral complications, including altered mental state, stroke, and seizure in 64 patients (3.8\%) [3]. Altered mental state occurred in 57 patients $(3.4 \%)$, including delirium and hypoxic-metabolic encephalopathy. Cerebral infarction occurred in 13
$(0.8 \%)$. The mortality rate in patients with a neurological complication was $29 \%$.

In several recent studies, psychomotor performance has been evaluated carefully both preoperatively and postoperatively [5]. The results demonstrated clear deterioration of performance, most frequently among patients with gross neurological deficits.

Relatively little attention has been paid to the longterm neurological consequences of open heart surgery. It has been stated that, although detailed neurological examination discloses postoperative deficits in a high percentage of patients after coronary artery surgery, many of these deficits do not appear to impair longterm function grossly [5]. In a five-year follow-up study, neuropsychological and neurological performances were evaluated in 44 patients who had undergone open heart surgery for valve replacement [6]. A distinct interrelationship was found between the clinical outcome immediately after operation and the neuropsychological long-term course.

The cause of many of the neurological complications of open heart surgery has not been determined, but mounting evidence has pointed to ischemic events secondary to microemboli. Studies of cerebrospinal fluid levels of adenylate kinase, an enzymatic marker of ischemic neural damage, showed increased levels in more than $50 \%$ of patients after open heart operation [7]. Moreover, neuropsychological deterioration in these patients was correlated with increases in enzyme levels. Evidence linking microemboli to neurological complications has also come from two recent investigations. A transcranial Doppler ultrasound investigation provided evidence for microemboli in the middle cerebral artery during cardiopulmonary bypass [8]. The incidence was substantially higher in patients undergoing cardiopulmonary bypass with a bubble oxygenator than in those with a membrane oxygenator. In another study, the retinal microvasculature was examined with fluorescein angiography in 21 patients undergoing elective coronary operations 5 minutes before cardiopulmonary bypass was discontinued [9]. All 21 patients had retinal microvascular occlusions indicative of microembolism. In related studies in dogs that had undergone open heart surgery, retinal histological studies revealed intravascular platelet-fibrin microaggregates [9].

Many sources of microemboli during open heart surgery have been suggested in the literature, including dislodgement of atheromatous debris from the 
aorta; release of left ventricular thrombus during cardiac manipulation; and microembolism of air, fat, platelet aggregates, fibrin, or silicone $[1,4]$. Other causative factors include cerebral hypoperfusion due to low flow, hypotension, incorrect placement of a cannula, or cerebrovascular occlusive disease [4].

A study published in the current issue of this journal provides the results of a neuropathological investigation of 43 patients who died following a surgical procedure $[10]$. Of these, 5 underwent cardiopulmonary bypass, 2 underwent aortography, and the remainder had neither cardiac surgery nor aortography. Similar studies were performed on the central nervous system of 6 dogs that underwent recent cardiopulmonary bypass operations and 6 that were not subjected to cardiopulmonary bypass operations. An alkaline phosphatase histochemical staining technique was used on thick celloidin sections to study the brain microvasculature. Many small capillary and arteriolar dilatations (SCADs) were found in terminal arterioles and capillaries of 4 of the 5 patients and all of the 6 dogs that had recently undergone cardiopulmonary bypass. The 2 patients who had undergone proximal aortography had a small number of the same kinds of changes. The patients and dogs that had not undergone cardiopulmonary bypass had no SCADs. The SCADs were empty and the investigators speculate that they represent the sites of gas bubbles or fat emboli that had been removed by the solvents used in tissue processing. The authors speculate that these SCADs may well underlie the neurological defects seen in patients following cardiac surgery assisted by cardiopulmonary bypass procedures. The findings reported by the authors evidently had been missed in previous studies of the neuropathological changes after open heart operation. Since these changes were seen consistently in both humans and dogs that had undergone bypass procedures, and not in humans or dogs that had not undergone bypass, the changes appear to be linked to the procedures and probably occur commonly in people undergoing cardiopulmonary bypass.

The frequent occurrence of neurological complica- tions after open heart surgery clearly has a basis in organic neuropathology. The abnormalities detected in the study cited above may provide the neuropathological basis for many of the cerebral complications described previously. Efforts to determine the cause of the changes and to take corrective action are strongly indicated.

Sid Gilman, MD

Department of Neurology

The University of Michigan

Ann Arbor, MI

\section{References}

1. Gilman S. Cerebral disorders after open-heart operations. N Engl J Med 1965;272:489-498

2. Slogoff S, Girgis KZ, Keats AS. Etiologic factors in neuropsychiatric complications associated with cardiopulmonary bypass. Anesth Analg 1982;61:903-911

3. Coffey CE, Massey EW, Roberts KB, et al. Natural history of cerebral complications of coronary artery bypass graft surgery. Neurology 1983;33:1416-1421

4. Shaw PJ, Bates D, Cartiidge NEF, et al. Early neurological complications of coronary artery bypass surgery. Br Med J 1985; 291:1384-1387

5. Prough DS, Stump DA, Troost BT. $\mathrm{PaCO}_{2}$ management during cardiopulmonary bypass: intriguing physiologic rationale, convincing clinical data, evolving hypothesis? Anesthesiology 1990;72:3-6

6. Sotaniemi KA, Mononen MA, Hokkanen TE. Long-term cerebral outcome after open-heart surgery. A five-year neuropsychological follow-up study. Stroke 1986;17:410-416

7. Aberg $T$, Ronquist $G$, Tyden $H$, et al. Adverse effects on the brain in cardiac operations as assessed by biochemical, psychometric, and radiologic methods. I Thorac Cardiovasc Surg 1984;87:99-105

8. Padayachee TS, Parsons S, Theobold R, et al. The detection of microemboli in the middle cerebral artery during cardiopulmonary bypass: a transcranial Doppler ultrasound investigation using membrane and bubble oxygenators. Ann Thorac Surg $1987 ; 44: 298-302$

9. Blauth CI, Arnold JV, Schulenberg WE, et al. Cerebral microembolism during cardiopulmonary bypass. I Thorac Cardiovasc Surg 1988;95:668-676

10. Moody DM, Bell MA, Challa VR, et al. Brain microemboli during cardiac surgery or aortography. Ann Neurol 1990;28: $477-486$ 